\title{
EFEITOS DA CONTAMINAÇÃO DA ÁGUA PELO FÁRMACO 17A-ETINILESTRADIOL, DETECÇÃO E TIPOS DE TRATAMENTO
}

\author{
Lima $\mathrm{PR}^{*}$, Bergamasco $\mathrm{R}^{* *}$
}

\begin{abstract}
Resumo
Assim como outros esteroides estrogênicos, como o estrona $\left(E_{1}\right)$, o $17 \beta$-estradiol $\left(E_{2}\right)$, e o estriol $\left(E_{3}\right)$, o 17 -etinilestradiol $\left(\mathrm{EE}_{2}\right)$ é um hormônio sintético, derivado do hormônio natural, que pode ser lançado no meio ambiente por meio de descarga de esgoto doméstico e de efluentes industriais não tratados. Ele é um dos medicamentos mais utilizados, o que aumenta seu potencial de presença no ambiente. Pode, também, contaminar o ambiente quando utilizado em atividades pecuárias e agrícolas. Este estudo centra-se em alertar acerca dos efeitos do $\mathrm{EE}_{2}$ à saúde humana e animal, bem como em informar que diversas pesquisas apontam a ineficácia de tratamento desse fármaco pelas Estações de Tratamento de Águas Residuais (ETARs), bem como pelas Estações de Tratamento de Efluentes (ETEs). Os mecanismos e alguns métodos de tratamento de águas contaminadas com o $\mathrm{EE}_{2}$ são discutidos sem desconsiderar alguns outros esteroides do mesmo grupo etinil.

Palavras-chave: 17a-etinilestradiol. Fármacos. Contaminação. Água. Tratamento.

* Pós-graduado em Biotecnologia Ambiental pela Universidade Estadual de Maringá; Graduado em Engenharia Ambiental pela Faculdade Oswaldo Cruz; mestrando em Biotecnologia Ambiental pela Universidade Estadual de Maringá; paulorenatolima@yahoo. com.br

${ }^{* *}$ Doutora em Engenharia Química pela Universidade Estadual de Campinas; Mestrado em Ciências de Alimentos pela Universidade Estadual de Londrina; ro.bergamasco@hotmail.com
\end{abstract}




\title{
Effects of water contamination by the 17a-etinylstradiol drug, detection and types of treatment
}

\begin{abstract}
Like other estrogenic steroids, such as estrone (E1), 17 $\beta$-estradiol (E2), and estriol (E3), 17 $\alpha$-ethinyl estradiol (EE2) is a synthetic hormone derived from the natural hormone that can be disposal of domestic sewage and untreated industrial effluent. It is one of the most commonly used drugs, which increases its potential for presence in the environment. It can also contaminate the environment when used in livestock and agricultural activities. This study focuses on the effects of EE2 on human and animal health, as well as informing that several studies point to the ineffectiveness of treatment of this drug by Wastewater Treatment Plants (WWTPs), as well as Effluents (ETEs). The mechanisms and some methods of treatment of water contaminated with EE2 are discussed without disregarding some other steroids of the same ethinyl group.

Keywords: $17 \alpha$-ethinylestradiol. Drugs. Contamination. Water. Treatment.
\end{abstract}

\section{INTRODUÇÃO}

Qualquer abordagem acerca da questão da água, escassez ou contaminação, possui intrinsecamente uma noção universal, uma vez que abrange as esferas ambientais, sociais, políticas e econômicas. Tratar dessa questão possui hoje grande relevância e sempre será assim, uma vez que é o elemento básico da vida e, portanto, universal.

Destaca-se, para efeito deste estudo, o grupo conhecido como desreguladores endócrinos (DEs), que constituem substâncias exógenas cuja capacidade de alterar as funções do sistema endócrino é alta, podendo, com isso, gerar riscos à saúde humana e animal, em especial aos peixes, os quais servem como fortes indicadores dos possíveis efeitos gerados em detrimento da desregulação endócrina. ${ }^{1,2}$

Os produtos químicos são conhecidos como compostos perturbadores endócrinos (em inglês, Endocrine Distupting Compounds - EDCs) e consistem em substâncias químicas que têm a capacidade de interferir na função normal dos sistemas endócrinos de seres humanos e de animais. Sendo, portanto, biotransformadores, os EDCs são alvo de muitas pesquisas científicas. ${ }^{3}$

A atenção neste estudo está voltada para o estrogênio sintético $17 \alpha$-etinilestradiol (ou $\mathrm{EE}_{2}$ ) por ser "o principal componente estrogênico utilizado em formulações de contraceptivos orais e um dos medicamentos mais consumidos no mundo", sendo ele "apontado como o principal composto responsável por provocar alterações endócrinas nos organismos aquáticos", ${ }^{4}$ motivos que preocupam a comunidade científica ao redor do mundo. $\mathrm{O}$ principal objeto das pesquisas em torno do $\mathrm{EE}_{2}$ se volta para o aspecto prejudicial da feminização anatômica da biota, comprometendo todo o sistema reprodutivo, gerando diversos problemas ecossistêmicos, ${ }^{5,6,7}$ todavia, estrona $\left(E_{1}\right), 17 \beta$-estradiol $\left(E_{2}\right)$ e estriol $\left(\mathrm{E}_{3}\right)$ não serão postos de lado. 
Neste estudo visou-se proceder ao levantamento de pesquisas sobre a contaminação do fármaco 17a-etinilestradiol em corpos d’água, bem como quais são os principais afetados por seus efeitos, os tipos de contaminantes e os seus respectivos graus de concentração e incidência, bem como os tipos de tratamento.

Não há ainda pesquisas que comprovem os efeitos de alterações endócrinas que levam à feminização masculina sobre a saúde humana no que se refere à exposição ou consumo de líquidos que contenham traços de $17 \alpha$-etinilestradiol. Por um lado, isso "em parte pode ser atribuído à acentuada dificuldade em demonstrar relação de causalidade quando se aborda o processo de desregulação en-

dócrina” ${ }^{2}$ e, por outro, pelo fator ético que pode envolver questões políticas referentes à feminização masculina.

Além da determinação do sexo e do atraso da maturidade sexual, o $\mathrm{EE}_{2}$ pode diminuir as características sexuais secundárias mesmo em baixas concentrações (ng/L), ${ }^{6}$ especialmente em indivíduos mais sensíveis à exposição, nos quais o hormônio pode provocar fortes alterações fisiológicas em virtude da desregulação endócrina.

Estudos mostram que em seres humanos a eficácia da ligação de $\mathrm{EE}_{2}$ ao receptor de estrogênio $(\mathrm{RE})$ é de uma a duas vezes maior do que de $\mathrm{E}_{2}$, demostrando ser até cinco vezes maior em algumas espécies de peixes. Essa maior afinidade do receptor indica que $\mathrm{EE}_{2}$ pode ser um composto estrogênico mais potente em termos de induzir uma resposta estrogênica em comparação ao $\mathrm{E}_{2}$ produzido naturalmente, embora as concentrações observadas pelos pesquisadores fossem extremamente baixas (ng/L) quando comparadas a outros contaminantes orgânicos antropogênicos. ${ }^{6}$

As duas vias básicas de contaminação ambiental dos corpos hídricos ocorre pela excreção ${ }^{6} \mathrm{e}$ pelo descarte, considerando que estudos apontam o $\mathrm{EE}_{2}$ como um dos principais fármacos contaminantes da água também no Brasil, cuja concentração média é de $5,8 \mu \mathrm{g} \mathrm{L}^{-1}$ no esgoto bruto do País. ${ }^{7}$ Isso ocorre, em especial, pelo fato de que "os atuais processos de tratamento de esgotos são incapazes de removê-los, sendo uma parte descartada em corpos receptores", 8 o que é corroborado por diversas outras pesquisas. ${ }^{6,9,10}$

Em razão de sua alta resistência ao processo de degradação, o $\mathrm{EE}_{2}$ tem ganhado cada vez mais atenção, considerando também sua tendência a absorver matéria orgânica, acumular-se em sedimentos e concentrar-se em biota. ${ }^{11}$

\section{MATERIAIS E MÉTODOS}

Utilizaram-se diversos artigos que relacionam a problemática do lançamento de $\mathrm{EE}_{2}$ em corpos hídricos por meio dos principais sites de busca de artigos científicos, quais sejam: PubMed, Capes, Scielo, ScienceDirect, Springer e Plos, os quais possuem maior relevância também na área de Biotecnologia Ambiental. O uso de palavras-chave como 17a-etinilestradiol, lançamento, fármacos, contaminação, água e tratamento ocorreu em larga medida, porém na língua inglesa, aumentando a 
amplitude, a relevância e a profundidade da pesquisa, a qual teve seus primeiros esboços em meados de 2016, mas alcançou sua maior profundidade no segundo semestre de 2017.

\section{O PROBLEMA DO LANÇAMENTO DE EE 2 EM CORPOS D’ÁGUA}

Os primeiros estudos para detecção de presença de fármacos no ambiente foram feitos nos Estados Unidos e datam do final da década de 1970. Detectou-se a presença de ácido clorídrico, metabólito dos antilipêmicos clofibrato, bem como de etofibrato. Identificou-se, ainda, que a principal fonte de lançamento acontecia por meio de esgotos domésticos e não mais por outras vias como se acreditava. Mesmo com isso, as indústrias farmacêuticas e os setores rurais também são considerados em diversas pesquisas como grandes lançadores indevidos de fármacos. ${ }^{7,12}$

Como é notório, os processos atuais de tratamento de água e esgoto não demonstram alta eficiência na detecção e remoção de diversos fármacos, o que inclui o 17 a-etinilestradiol. O principal fator ocorre em razão de as estações possuírem ação biocida ou estruturas químicas complexas não passíveis de biodegradação, comprovado por diversos estudos que mostram a presença desse tipo de contaminante em corpos hídricos. Sendo a biodegradação o principal mecanismo de remoção de estrogênios esteroides em Estações de Tratamento de Efluentes (ETEs) ${ }^{13,14}$ e Estações de Tratamento de Águas Residuais (ETARs).

$\mathrm{O} \mathrm{EE}_{2}$ aparece no Brasil como um dos principais problemas, sendo liberado em corpos hídricos como um xenoestrógeno, causando sérios problemas ao sistema endócrino humano e animal. A Figura 1 apresenta sua fórmula química estrutural.

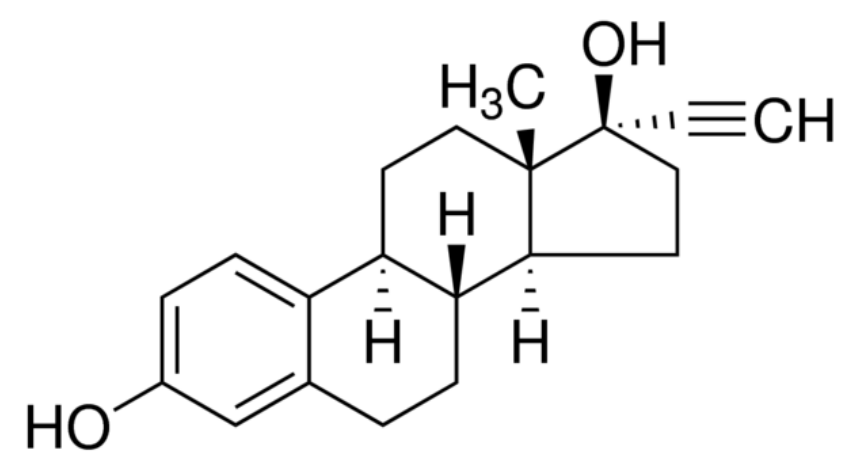

Figura 1 - E4876 SIGMA 17 -ethynylestradiol $\geq 98 \%\left(\mathrm{C}_{20} \mathrm{H}_{24} \mathrm{O}_{2}\right)$

Nota: Peso molecular: $296.40 \mathrm{amu}$

Fonte: Lee e Liu. ${ }^{14}$

Pode-se ver a seguir as concentrações médias de alguns fármacos detectados em ambientes aquáticos, considerando que o único fármaco que possui concentração superior ao 17a-etinilestradiol é o ibuprofeno: 
Tabela 1 - Concentrações médias de fármacos detectados em ambientes aquáticos (elementos com maior grau de significância)

\begin{tabular}{lrll}
\hline \multicolumn{1}{c}{ Fármaco (classe terapêutica) } & Concentração média $(\boldsymbol{\mu g}$ L-1) & Matriz \\
\hline Diclofenaco (antiinflamatório) & 2,9 & Esgoto bruto/Brasil \\
& 4,0 & Água superficial/Brasil \\
17a-etinilestradiol (hormônio contraceptivo) & 5,8 & Esgoto bruto/Brasil \\
Ibuprofeno (antiinflamatório) & $13,1 \quad$ Esgoto bruto/Finlândia \\
Ibuprofeno (antiinflamatório) & 54,2 & Esgoto bruto/Brasil \\
Naproxeno (antiinflamatório) & 4,9 & Esgoto bruto/Finlândia \\
\hline
\end{tabular}

Fonte: Melo, Travó, Bautitz e Nogueira. ${ }^{7}$

Com efeito, o $\mathrm{EE}_{2}$ também pode ser considerado um Agente Hormonalmente Ativo (AHA). Os AHAs consistem em um grupo especial de contaminantes orgânicos que afetam os sistemas nervoso, endócrino e imunológico dos seres vivos e são capazes de perturbar o sistema endócrino ao bloquear ou imitar um hormônio, além de serem referidos como produtos químicos hormonalmente ativos ou disruptores endócrinos. Atualmente, os seres vivos são diretamente afetados na expressão gênica em níveis extremamente baixos (como $0,2 \mathrm{mg} / \mathrm{L}^{-1} \mathrm{de} \mathrm{EE}_{2}$ ), tanto genética quanto epigeneticamente, prejudicando, assim, as formas de vida individuais ou na prole ou população. Isso pode acarretar diversas doenças também em humanos, como cânceres, deficiências de desenvolvimento e neurológicas, disfunção de glândulas e de fertilidade, entre outros males. ${ }^{16}$

\subsection{MALEFÍCIOS AOS ANIMAIS}

No que se refere aos malefícios aos animais, o peixe possui maior atenção dos pesquisadores e revisores. No ser humano, "a principal referência a hormônios sintéticos recai sobre o 17a-etinilestradiol ou etinilestradiol (EE2), fármaco amplamente utilizado em pílulas anticoncepcionais." ${ }^{77}$

\footnotetext{
No estudo de Routledge et al., duas espécies de peixes, Oncorhynchus mykiss e Rutilus rutilus, foram expostas por 21 dias a concentrações de 17b-estradiol e estrona ambientalmente relevantes $\left(1,10,100 \mathrm{ng} \mathrm{L}^{-1}\right)$. De acordo com esses e outros pesquisadores, os resultados confirmaram que os estrogênios identificados em efluentes domésticos estão presentes em quantidades suficientes para induzir a síntese de VTG em espécies de peixes. Efluentes de ETE vêm sendo apontados como a maior causa de efeitos estrogênicos em peixes. ${ }^{1}$
}

Os produtos químicos perturbadores endócrinos (EDCs), como é o caso dos fármacos supracitados, comprometem o desenvolvimento sexual e neural das espécies de peixes, considerando o relato de diversos casos de alterações endócrinas de machos para fêmeas. Mesmo não alterando diretamente o sexo sob a ótica fenotípica, existem indícios de que vários EDCs podem desmasculinizar comportamentos específicos do sexo masculino que são essenciais para atrair um companheiro. $\mathrm{O}$ 
problema, além de reduzir a probabilidade da reprodução, é a de alterar significativamente o epigenoma do esperma, causando problemas transgeneracionais. ${ }^{18}$

Ademais, há diversos outros estudos que concentram sua ótica na análise do perfil de feminização de expressão de genes que são alterados pela exposição ao etinilestradiol, ${ }^{6,18,19,20}$ como do peixe de lixívia (Protosalanx hyalocranius). Os resultados destes estudos mostraram que a exposição contínua dos embriões/larvas de peixe a altas concentrações de $\mathrm{E} 2$ e EE 2 implicou um aumento drástico da mortandade dos peixes após o período de 17 dias. De acordo com as pesquisas, uma concentração de E2 de $8 \mu \mathrm{g} . \mathrm{L}^{-1}$ (dia 16) e uma concentração de $\mathrm{EE}_{2}$ de $0,2 \mathrm{mg} \cdot \mathrm{L}^{-1}$ (dia 18) induziram taxas de teratogênese máxima de $30 \%$ e $35 \%$, respectivamente. E, embora as concentrações de $\mathrm{E}_{2}$ e $\mathrm{EE}_{2}$ ambientalmente relevantes não sejam letais para embriões ou larvas de $P$. hyalocranius, sua capacidade de induzir teratogênese e retardo de incubação é motivo de forte preocupação. ${ }^{21}$

A remoção da ecotoxicidade do $\mathrm{EE}_{2}$ utilizando o tratamento com água TAML/peróxido (Oxidative Degradation of Metaldehyde: Pursuing Better Water Treatment for the Most Persistent), por exemplo, restringe a estrogenicidade in vitro e diminui substancialmente a feminização dos peixes in vivo. ${ }^{6}$

Mesmo sendo nos peixes o caráter analítico substancial das pesquisas, há também olhares que se voltam para a análise da presença de estrogênios nos excrementos de gado, considerando que a primeira investigação para remover simultaneamente os estrogênios foi feita em 2016 - as pesquisas incluíram o estriol ( $\left.\mathrm{E}_{3}\right)$, bisfenol A (BPA), dietilstilbestrol (DES), estradiol ( $\left.\mathrm{E}_{2}\right)$ e etinilestradiol ( $\left.\mathrm{EE}_{2}\right)$, a partir de estrume de vaca utilizando uma técnica de oxidação de Fenton. ${ }^{22}$

Há, ainda, uma pesquisa voltada para o estudo dos efeitos do $\mathrm{EE}_{2}$ em ratos, demonstrando que a exposição ao benzo(a)pireno ( $\mathrm{BaP})$, que também é um disruptor endócrino, assim como o EE2, induz à expressão da proteína CYP1A1 e sua atividade marcadora no fígado, rim e pulmão desses animais. ${ }^{23}$

Os métodos utilizados pelos pesquisadores para avaliar as alterações na expressão da proteína citocromo $\mathrm{P} 450$ (CYP) após a exposição de ratos ao benzo(a)pireno (BaP) e $\mathrm{EE}_{2}$ foram analisados por Western Blot. No que se refere à análise das atividades específicas de CYP1A1, 2C e 3A em microssomas hepáticos isolados de ratos expostos, utiliza-se a cromatografia líquida de alta eficiência (HPLC). A expressão da proteína CYP2C11 em contato com o $\mathrm{EE}_{2}$ aumenta no fígado dos ratos e diminui no pulmão. A atividade da proteína CYP2C6 também aumenta quando o $\mathrm{EE}_{2}$ é administrado individualmente. Não se encontra efeito ou mesmo diminuição na expressão de CYP3A. ${ }^{23}$

A análise cromatográfica na pesquisa de Daniel e Lima foi realizada em modo isocrático, na fase móvel 50\% ACN: $\mathrm{H}_{2} \mathrm{O}$ pH 3,0; coluna C18, vazão $1 \mathrm{~mL} \cdot \mathrm{min}^{-1}$, injeção $10 \mu \mathrm{L}, \lambda=281 \mathrm{~nm}, \mathrm{~T}=40$ C. Os limites de quantificação que os autores encontraram foram de $3,75 \mu \mathrm{gL}^{-1}$ para o $17 \alpha$-etinilestradiol e de $7,75 \mu \mathrm{gL}^{-1}$ para o estriol, enquanto os de detecção foram de $1,12 \mu \mathrm{gL}^{-1}$ para o 17 a-etinilestradiol e de $2,32 \mu \mathrm{gL} \mathrm{L}^{-1}$ para o estriol. ${ }^{8}$

A precisão avaliada pelos pesquisadores nos quatro diferentes níveis de concentração apresentou coeficiente de variância (\%CV) e exatidão inferiores a 3\%. Os testes de extração SPE demonstraram que o "condicionamento utilizando-se de acetonitrila, eluição com metanol mostrou valores de recuperação superiores a 75\%." Tal metodologia apresentou adequada determinação de hormônios estrógenos em águas naturais. ${ }^{8}$ 


\subsection{MALEFÍCIOS AOS HUMANOS}

É evidente que a contaminação de animais pode trazer efeitos adversos à saúde humana, especialmente no que se refere ao consumo de proteínas, que é feito por meio da alimentação com carnes, como peixe e gado.

Entretanto, os seres humanos também podem ser expostos ao $\mathrm{EE}_{2}$ por meio do consumo de água potável. Não somente o $\mathrm{EE}_{2}$, mas os demais esteroides estrogênicos mencionados na Figura $2\left(\mathrm{E}_{1}\right.$, $\mathrm{E}_{2} \mathrm{e}_{3}$ ) são relatados como contaminantes de água potável por diversos outros estudos. ${ }^{24,25}$<smiles>CC12CCC3c4ccc(O)cc4CCC3C1CCC2O</smiles>

$17 \beta$-Estradiol $\left(\mathrm{E}_{2}\right)$<smiles>CC12CCC3c4ccc(O)cc4CCC3C1CCC2=O</smiles>

Estrone $\left(E_{1}\right)$<smiles>CC12CCC3c4ccc(O)cc4CCC3C1CC(O)C2O</smiles>

Estriol $\left(\mathrm{E}_{3}\right)$

Figura 2 - Estruturas químicas de $17 \beta$-estradiol

Fonte: Lee e Liu. ${ }^{14}$

Como principais transformações dos estrogênios esteroides, a degradação pode ser mediada microbiologicamente pela quebra de sulfatos e glicuronídeos pelas bactérias coliformes fecais (enzima glucuronidase de E. coli), bem como pela oxidação de $\mathrm{E}_{2}$ e $\mathrm{EE}_{2} \mathrm{e}_{1}{ }_{1}^{13,14}$ A seguir é possível verificar o processo de degradação por micro-organismos do $\mathrm{E}_{2}$ e $\mathrm{E}_{1}$ (Figura 3).

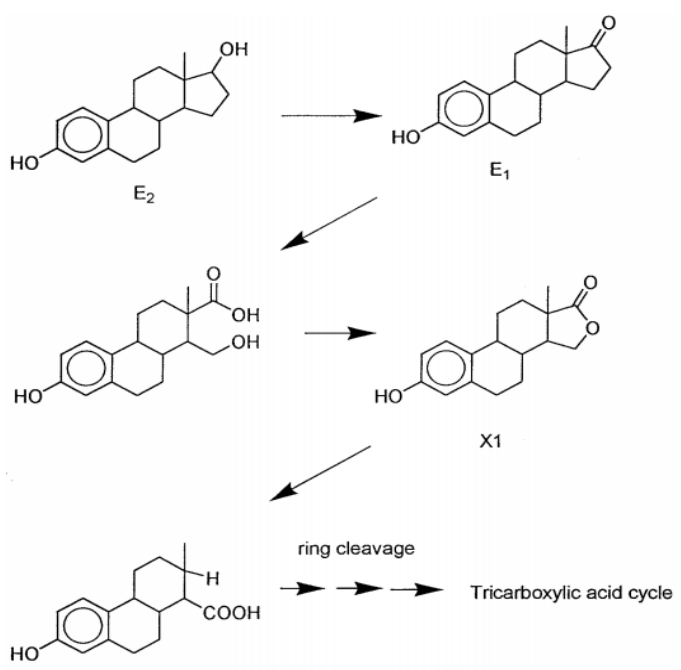

Figura 3 - Via metabólica de E2 e E1 por micro-organismos em águas residuais Fonte: Lee e Liu. ${ }^{14}$ 
Considerando $\mathrm{o} \mathrm{EE}_{2}$ é o hormônio mais difícil para se degradar, sob o aspecto biológico, este demonstrou ser o mais persistente à biodegradação. Isso ocorre em razão de que o $\mathrm{E}_{2}$ pode combater quantitativamente o $\mathrm{E}_{1}$, mas é incapaz de combater o próprio $\mathrm{EE}_{2}{ }^{26}$ Ademais, há uma mineralização de $20 \%$ para $\mathrm{EE}_{2}$ e $75 \%$ para $\mathrm{E}_{2}$ em $24 \mathrm{~h}$. Tal descoberta pode ser atribuída ao grupo etinil do $\mathrm{EE}_{2}$, o que dificulta em grande medida a expressão enzimática, bem como a ligação do substrato-receptor e com o metabolismo. ${ }^{27}$

De acordo com os mesmos estudos, a biodegradação de estrogênios esteroides naturais é mais eficiente sob condição aeróbica do que anaeróbica, o que inclui o $\mathrm{EE}_{2}$, considerando que a temperatura mais elevada no tratamento melhora a eficiência microbiana. ${ }^{28}$

\section{DETECÇÃO DO FÁRMACO $\left(\mathrm{EE}_{2}\right)$}

A "principal via de contaminação do meio ambiente com fármacos e desreguladores endócrinos (DE) é o lançamento de esgotos in natura e tratado." ${ }^{17}$ Desse modo, estando a Escherichia coli associada à contaminação da água por material fecal lançado por seres humanos e animais, ela passa a ser considerada uma boa indicadora para a qualidade da água. Em outras palavras, a E. Coli ajuda na detecção de alta ou baixa contaminação de fármacos no ambiente. ${ }^{29}$

Há também o método de detecção que ocorre por meio de um procedimento voltamétrico, validado como uma ferramenta de triagem. Assim, detecta-se o $\mathrm{EE}_{2}$ em amostras de água sem etapas de extração, limpeza ou derivatização. ${ }^{30}$ É possível detectá-lo também por espectrometria, metodologia disponível em laboratório.

A detecção de $\mathrm{EE}_{2}$ em amostras ambientais, formulações farmacêuticas e em amostras biológicas tem sido realizada por várias técnicas analíticas, incluindo, portanto, espectrometria líquida e cromatografia em fase gasosa, eletroforese, imunoensaios e eletroquímica. E, ainda, outros métodos largamente utilizados para determinar $\mathrm{EE}_{2} \mathrm{em}$ amostras ambientais são baseados em técnicas cromatográficas, como cromatografia líquida de alta eficiência (HPLC), cromatografia líquida (LC) e espectrometria de massa-cromatografia líquida (LC/MS).

As técnicas eletroanalíticas são tidas como uma nova proposta de detecção, mais rápidas e, ao que parece, mais eficientes. Elas permitem a análise rápida cujas vantagens incluem a capacidade de triagem, de alta sensibilidade, facilidade de operação, o potencial de miniaturização e medição in situ, baixo custo, bem como liberdade de tratamento da amostra e pré-concentração. Ademais, as técnicas voltamétricas não requerem o uso de solventes orgânicos tóxicos. ${ }^{30}$

Os resultados dos pesquisadores indicaram que o rastreio hormonal pode ser realizado sem a necessidade de tratamento prévio da amostra (extração ou limpeza). Com efeito, a voltametria em um eletrodo de mercúrio demonstrou ser um procedimento viável para a análise dos traços de compostos orgânicos. $\mathrm{O} \mathrm{EE}_{2}$ pode ser quantificado com maior precisão em amostras aquosas utilizando o HMDE (eletrodo de gota de mercúrio suspensa). ${ }^{30}$ 
Ainda há diversas outras metodologias analíticas que são utilizadas, como por exemplo, a fluorescência, a espectrometria de massas e a análise por cromatografia líquida acoplada a detectores de arranjo de diodos. A utilização da metodologia cromatográfica a gás não é tão comum, uma vez que "as amostras precisam passar pelo processo de derivatização para que sejam analisadas." ${ }^{31}$ Enquanto a "utilização de cromatografia líquida de alta eficiência acoplada a detector de espectrometria de massas é altamente precisa e sensível, sendo uma das mais indicadas para análise de fármacos." ${ }^{31}$ Algo que pode ser pensado como melhor solução dos problemas causados pela descarga de desreguladores endócrinos no ambiente aquático, trata-se de "uma reformulação nos meios de tratamento tradicionais, como utilização da ozonização e por raios UV." ${ }^{31}$

Em trabalho ${ }^{8}$ que empregou a extração em fase sólida (SPE), fez-se uso de cartuchos adsorventes de octadecil sílica (C18), bem como análise por HPLC a fim de determinar a presença de estriol, $\beta$-estradiol, $17 \alpha$-etinilestradiol e estrona na água.

\section{TIPOS DE TRATAMENTO}

É difícil controlar o lançamento de compostos estrogênicos na água por meio de políticas rigorosas, como a proibição da produção de hormônios sintéticos, em especial pela importância na regulação do sistema endócrino. Destarte, os efeitos fisiológicos e os possíveis impactos negativos dos estrogênios sintéticos no ambiente podem ser minimizados por meio de vários tratamentos e procedimentos de remediação. ${ }^{6}$

A pesquisa que faz uso de nanopartículas de prata (AgNPs), as quais possuem atividade antibacteriana e citotoxicidade - que são resultantes do cátion de prata dissolvido $\left(\mathrm{Ag}^{+}\right)$e de seus complexos solúveis -, demonstra que essas partículas podem causar a oxidação in situ de contaminantes emergentes em meio ambiente aquoso quando na presença do $\mathrm{EE}_{2}{ }^{32}$

Técnicas de caracterização, como análise elementar, espectroscopia de infravermelho médio (FT-IR), análise termogravimétrica (TGA) e microscopia eletrônica de varredura (MEV), podem ser consideradas pelos pesquisadores que trabalham com o tratamento do $\mathrm{EE}_{2}$, o que inclui a possibilidade da investigação da capacidade de adsorção da Moringa oleífera para a remoção de $\mathrm{EE}_{2}$.

Com isso, o estudo de isotermas de adsorção, o qual é influenciado pela massa de adsorvente na adsorção, influencia, por sua vez, o pH na remoção dos compostos, bem como o tempo de equilíbrio quando em solução. A análise pelo método de Brunauer, Emmett e Teller (BET), conhecido também por Teoria de Adsorção Multimolecular, e a análise granulométrica poderão ser úteis nos estudos de remoção do fármaco objeto deste artigo. 


\subsection{NANOPARTÍCULAS E DEGRADAÇÃO POR OXIDAÇÃO}

Em pesquisas ${ }^{33}$ que utilizaram as propriedades catalíticas das nanopartículas por meio de eliminadores de radicais, avaliaram-se os produtos de transformação de $\mathrm{EE}_{2}$. Elas demonstraram que, sob a redução direta do $\mathrm{EE}_{2}$ por nZVI (nano zero valent iron), ocorre maior degradação em todos os pHs. Após a purga de ar, $\mathrm{o} \mathrm{EE}_{2}$ apresenta as mesmas espécies radicais que o sistema não purgado no mesmo $\mathrm{pH}$, contudo a taxa de degradação é menor com mais oxigênio. Os radicais dominantes são: $\mathrm{OH}$ a $\mathrm{pH} 3$ e $\mathrm{O}_{2}$ a $\mathrm{pH} 5$, e, embora nenhum dos radicais estivesse envolvido no $\mathrm{pH}$ 7, os pesquisadores afirmam que nenhuma conclusão poder ser feita acerca de qual radical realmente está envolvido no $\mathrm{pH} 7 .^{33}$

Há, ainda, outra pesquisa em que dois experimentos independentes, a saber: sorção e oxidação não específica de medicação oxigenada de estrogênios, confirmaram uma diminuição significativa da concentração de estrogênios quando são aplicados pelo menos $2 \mathrm{~g} \mathrm{~L}^{-1}$ de nZVI. O estudo apresentado fornece informações sobre os mecanismos de interação nZVI com estrogênios esteroides sob condições aeróbicas prevalentes nas tecnologias de tratamento de água. ${ }^{34}$

É possível avaliar a remoção de $\mathrm{EE}_{2}$ com soluções-estoque de etinilestradiol preparadas a partir do composto puro e de pílulas anticoncepcionais e, em seguida, diluídas para preparar soluções de trabalho. A turbidez da água, geralmente, não se apresenta como fator fortemente relevante no tratamento de águas contaminadas com o fármaco $\mathrm{EE}_{2 .}{ }^{29}$

Park et al. ${ }^{32}$ utilizaram nanopartículas de prata (AgNPs) para oxidação in situ de contaminantes fármacos. Existe também a possibilidade da utilização de partículas de carvão funcionalizado com grafeno e prata para replicar em partes esse trabalho desenvolvido. ${ }^{32}$

Os pesquisadores fazem uso de produtos químicos e de qualidade analítica. A metodologia empregada utiliza-se das AgNPs revestidas com PVP, com um tamanho de partícula especificado pelo fabricante $<100 \mathrm{~nm}, \mathrm{H}_{2} \mathrm{O}_{2}$ e soluções tampão-padrão, cuja pureza pode ser superior a 99,5\%. Os resultados indicam que a degradação AgNPs mediada com concentrações relativamente baixas de $\mathrm{H}_{2} \mathrm{O}_{2}$ é altamente eficaz na remoção de $\mathrm{BPA}$ e $\mathrm{EE}_{2}$ sob condições ácidas. $\mathrm{O}$ pH da solução e a concentração de $\mathrm{H}_{2} \mathrm{O}_{2}$ têm efeitos significativos nos tamanhos das AgNPs e no desempenho na degradação de endocrine disrupting chemicals (EDCs), ou seja, trata-se de produtos químicos que perturbam o sistema endócrino, indicando que a geração de $\mathrm{OH}$ durante a dissolução catalítica de AgNPs pode ser amplificada ajustando o pH e a concentração de $\mathrm{H}_{2} \mathrm{O}_{2}{ }^{32}$

Os próprios pesquisadores ${ }^{32}$ destacam que lideram o caminho para a identificação do potencial impacto da copresença de outras nanopartículas no setor de engenharia química e biotecnológica, por exemplo, as nanopartículas de ferro zero-valente [nZVI], em sistemas de AgNPs somados ao $\mathrm{H}_{2} \mathrm{O}_{2}$ na degradação oxidativa de contaminantes orgânicos.

As nanopartículas de prata (AgNPs) foram relatadas como tendo atividades antibacterianas e citotoxicidade, resultantes do cátion de prata dissolvido (Agp) e seus complexos solúveis. Por ser o primeiro relatório sobre o assunto, examina-se a eficácia da degradação oxidativa de bisfenol A (BPA) e do 17 a-etinilestradiol (EE2) em água por espécies que reagem ao oxigênio e que são formadas duran- 
te a decomposição de $\mathrm{H}_{2} \mathrm{O}_{2}$, auxiliadas pelo polímero polivinilpirrolidona (PVP) AgNPs. A cinética de oxidação de BPA e EE2 por PVP-AgNPs pode ser interpretada usando três abordagens de modelagem diferentes, de acordo, ainda, com os estudos desses pesquisadores, quais sejam: uma pseudo-primeira ordem inicial; uma taxa de primeira ordem; e a equação cinética de Behnajady-Modirshahla-Ghanbery. Os resultados mostram que as AgNPs podem ter potencial para facilitar a oxidação in situ de contaminantes emergentes no meio aquoso. ${ }^{32}$

Acerca dessa degradação, é esperado que, conforme resultados da pesquisa supracitada, a reação de degradação catalisada por nanopartículas não possa mineralizar completamente os EDCs, mas, sim, formar produtos de transformação ou intermediários halogenados na presença de brometo, que são frequentemente pouco biodegradáveis em água. Destarte, a importância de pesquisas que prossigam na análise desses estudos, poderá propor soluções talvez mais completas.

\subsection{FOTOCATÁLISE E OXIDAÇÃO}

A remediação da solução aquosa de 17 a-etinilestradiol pode ser feita também pelo processo de fotocatálise assistida eletroquimicamente usando eletrodos $\mathrm{TiO}_{2}$ e $\mathrm{TiO}_{2} / \mathrm{WO}_{3}$ irradiados por um simulador solar. Os fotocatalisadores consistem em películas porosas de 4,5 $\mu \mathrm{m}$ de espessura de $\mathrm{TiO}_{2}$ e $\mathrm{TiO}_{2} / \mathrm{WO} 3$ (razão molar W/Ti de 12\%), depositadas em eletrodos transparentes a partir de suspensões aquosas de partículas de $\mathrm{TiO}_{2}$ e precursores de $\mathrm{WO} 3$, seguidas de tratamento térmico a $450{ }^{\circ} \mathrm{C} .{ }^{35}$

As soluções $\mathrm{EE}_{2}$ resolvidas foram analisadas por medições de fluorescência, HPLC e carbono orgânico total, e ambos promoveram a oxidação. $\mathrm{O} \mathrm{TiO}_{2} / \mathrm{WO} 3$ híbrido retém luz solar melhorada e separa melhor os transportadores de carga fotogenerados, resultando em maior desempenho na remoção do $\mathrm{EE}_{2}{ }^{35}$

Já a degradação de $\mathrm{EE}_{2}$ em solução aquática por oxidação eletrocatalítica aplicando o eletrodo $\mathrm{SnO}\left({ }_{2}\right) \mathrm{Ti} / \mathrm{SnO}\left({ }_{2}\right)$ baseado em $\mathrm{Ti}$, indica que as atividades estrogênicas dos produtos intermediários podem ser insignificantes. ${ }^{36}$

$\mathrm{O}$ processo de remoção de $\mathrm{EE}_{2}$ por um fotorreator modificado a partir de um conjunto de desinfecção ultravioleta que é desenvolvido com materiais $\mathrm{TiO}_{2} / \mathrm{FC}$ também foi investigado em outro estudo. ${ }^{37}$ Um modelo integrado, incluindo um modelo de espalhamento de adsorção de placas e um modelo de difusão de fluxo modificado, foi estabelecido para simular o processo de degradação fotocatalítica com diferentes campos de radiação, carga de contaminantes e velocidade de fluxo. Os resultados são satisfatórios no que se refere às simulações dos modelos e aos resultados experimentais, mostrando forte potencial para o projeto de ampliação do reator fotocatalítico modificado. ${ }^{37}$

\section{CENÁRIO HODIERNO DO LANÇAMENTO DE FÁRMACOS}

Infelizmente, é notório que o mundo passa por uma das maiores crises no abastecimento de água potável, própria para o consumo humano. A despeito da existência, por vezes rigorosas, das 
leis de saneamento, há cada vez menos acesso aos recursos hídricos de qualidade. Isso significa que sem a intervenção severa do próprio homem as condições de saúde no futuro estarão cada vez mais problemáticas. Em 2006, mais de 2,5 bilhões de pessoas não tinham acesso a instalações sanitárias. ${ }^{38}$

O World Water Development Report de 2003, da UNESCO-WWAP, estima "que em 2050 um quarto da população do planeta viverá em situação de escassez crônica de água potável”, sendo que, no Brasil, apenas "um terço dos 40\% mais pobres dispõem de serviços de água e saneamento, enquanto que para os $10 \%$ mais ricos esse valor sobe para $80 \% .{ }^{39}$ Se houver, portanto, problemas de abastecimento, o que dizer, então, se não houver técnicas limpas e baratas para tratar corretamente a pouca água disponível?

Logo, a ação antropológica está diretamente ligada às causas de fatores de baixa qualidade da água e às doenças adquiridas pelo consumo de água contaminada, uma vez que alteram física, química e biologicamente o ecossistema afetado, e, ainda, é possível incluir como agravante a má gestão dos recursos hídricos por parte das autoridades competentes e dos órgãos ambientais.

Com efeito, o lançamento de resquícios de medicamentos, sejam despejados de modo deliberado, sejam de modo fortuito, representam um problema potencial para o tratamento de águas, tanto nas Estações de Tratamento de Águas Residuais (ETARs) quanto nas Estações de Tratamento de Efluente (ETEs). Tal situação é agravada já que o 17a-etinilestradiol se apresenta como o mais persistente dos estrogênios, com um tempo de meia-vida em água de aproximadamente 17 dias e uma baixa taxa de fotodegradação. ${ }^{40}$

Para se discutir a legislação referente ao 17a-etinilestradiol, é preciso apresentar que há dois tipos de normas que se aplicam ao tema água, a saber: aquelas que têm como foco os critérios de qualidade da água recomendados para a proteção da vida aquática e outras que visam à proteção da saúde dos seres humanos que a ingerem. Infelizmente, no Brasil, não há legislação específica. Já nos Estados Unidos, a Contaminant Candidate List (CCL 3), publicada em 2009, inclui o $\mathrm{EE}_{2}$ na lista dos 112 contaminantes (12 microbiológicos e 100 químicos), no ano $2015 .^{4}$

Segundo Aquino, Brandt e Chernicharo, ${ }^{17}$ os "fármacos e DE podem atingir as redes de coleta de esgoto por meio do lançamento de águas cinzas" - como as dos chuveiros, lavatórios e lavanderias - que são lançadas contaminadas com medicamentos de uso oral e hormônios naturais - e descartes diversos. Com efeito, "as estações de tratamento de esgoto (ETE) normalmente empregam processos biológicos como principal tecnologia e, em poucos casos, utilizam técnicas complementares de tratamento." ${ }^{17}$ Por fim:

[...] as unidades da ETE são projetadas para reduzir a carga de poluentes orgânicos e, eventualmente, nutrientes e microrganismos patogênicos, não objetivando especificamente a remoção de fármacos e DE presentes no esgoto sanitário. Qualquer remoção desses compostos que possa ocorrer é fortuita e inerente ao processo de tratamento. ${ }^{17}$

Portanto, há forte potencial de transferência de medicamentos e produtos para cuidados pessoais (PPCPs) e hormônios esteroides de esgotamento sanitário para o meio ambiente. Os PPCPs e 
esteroides são encontrados em lagoas de tratamento de esgoto, não somente urbanas, mas também rurais, apontando claramente para a ineficácia dos sistemas de tratamento atuais, como tanques sépticos e de lagoas, demonstrando baixa eficácia na remoção de contaminantes e fazendo com que o efluente oriundo de lagoas arejadas também retenha metais pesados, patógenos e poluentes orgânicos, além de quantidades excessivas de nutrientes.

Essa universalização é o que garante a importância, a qual objetiva, entre outros fins, obter tecnologias biotecnológicas para o fornecimento de um saneamento básico de melhor qualidade, dada sua abrangência infraestrutural e operacional, bem como de abastecimento de água, esgotamento sanitário, limpeza urbana, manejo de resíduos sólidos e drenagem urbana.

Desse modo, uma revisão sobre a ocorrência, os mecanismos e o destino do $\mathrm{EE}_{2}$ lançados por vias domésticas (excreção humana) ou sintéticas/industriais em todos os sistemas de tratamento de águas residuais é crucial para determinar a remoção e a carga recebida desses compostos no meio ambiente.

\section{CONSIDERAÇÕES FINAIS}

Neste estudo visou-se discutir sobre a presença de resíduos do fármaco 17 -etinilestradiol no meio em que é lançado, bem como se consideraram as relações patológicas e toxicológicas dos efeitos desse fármaco, verificando-se que todas as pesquisas que envolvem o efeito de seu lançamento no meio o consideram um contaminante ambiental. Sugere-se que pesquisas futuras se concentrem na exposição e no potencial efeito tóxico do $\mathrm{EE}_{2}$ em humanos e em organismos terrestres diversos, pois os estudos existentes são irrelevantes em termos quantitativos. e em organismos terrestres diversos. As diferentes concentrações de $\mathrm{EE}_{2}$ têm diferentes efeitos tóxicos em diferentes espécies de organismos. Desse modo, mais estudos sobre bioconcentração, bioacumulação, transporte de cadeia alimentar, biotransformação, eliminação e distribuição de $\mathrm{EE}_{2}$ em organismos de diferentes níveis tróficos no meio ambiente são essenciais para a continuidade de pesquisas acerca de seus danos para a saúde da fauna, da flora e humana.

\section{REFERÊNCIAS}

1. Bila DM, Dezotti M. Desreguladores endócrinos no meio ambiente: efeitos e consequências. Quím. Nova. 2007;30:651-66.

2. Olivares-Rubio HF, Dzul-Caamal R, Gallegos-Rangel ME, Madera-Sandoval RL, Domínguez-López ML, García-Latorre E, et al. Relationship between biomarkers and endocrine-disrupting compounds in wild Girardnichthys viviparus from two lakes with different degrees of pollution. Ecotoxicology. 2015 Apr;24(3):664-85.

3. Liu J, Wang R, Huang B, Lin C, Zhou J, Pan X. Biological effects and bioaccumulation of steroidal and phenolic endocrine disrupting chemicals in high-back crucian carp exposed to wastewater treatment plant effluents. Environ. Pollut. 2012;162:325-31. 
4. Cunha DL, Silva SMC, Bila DM, Oliveira JLM, Sarcinelli PN, Larentis AL. Regulamentação do estrogênio sintético $17 \alpha$-etinilestradiol em matrizes aquáticas na Europa, Estados Unidos e Brasil. Cad. Saúde Pública. 2016 mar;32(3).

5. Bhandari RK, Deem SL, Holliday DK, Jandegian CM, Kassotis CD, Nagel SC, et al. Effects of the environmental estrogenic contaminants bisphenol A and 17a-ethinyl estradiol on sexual development and adult behaviors in aquatic wildlife species. Gen Comp Endocrinol. 2015 Apr;1(214):195219.

6. Aris AZ, Shamsuddin AS, Praveena SM. Occurrence of 17a-ethynylestradiol (EE2) in the environment and effect on exposed biota: a review. Environ Int. 2014 Aug;69:104-19.

7. Melo SAS, Trovó AG, Bautitz IR, Nogueira RFP. Degradação de fármacos residuais por processos oxidativos avançados. Quím. Nova. 2009;32(1):188-97.

8. Daniel MS, Lima EC. Determinação simultânea de estriol, $\beta$-estradiol, $17 \alpha$-etinilestradiol e estrona empregando-se extração em fase sólida (SPE) e cromatografia líquida de alta eficiência (HPLC). Rev. Ambient. Água. 2014 dez; 9(4):688-95.

9. Cong VH, Iwaya S, Sakakibara Y. Removal of estrogens by electrochemical oxidation process. J Environ Sci. 2014 Jun;26(6):1355-60.

10. Brandt EMF, Queiroz FB, Afonso RJCF, Aquino SF, Chernicharo CAL. Behaviour of pharmaceuticals and endocrine disrupting chemicals in simplified sewage treatment systems. J Environ Manage. 2013;128:718-26.

11. De Wit M, Keil D, van der Ven K, Vandamme S, Witter E, de Coen W. An integrated transcriptomic and proteomic approach characterizing estrogenic and metabolic effects of 17a-ethinylestradiol in zebrafish (Danio rerio). Gen Comp Endocrinol. 2010;167:190-210.

12. Crouse BA, Ghoshdastidar AJ, Tong AZ. The presence of acidic and neutral drugs in treated sewage effluents and receiving waters in the Cornwallis and Annapolis River watersheds and the Mill CoveSewage Treatment Plant in Nova Scotia, Canada. Environmental Research. 2012;112:92-9.

13. Manickum T, John W. Occurrence, fate and environmental risk assessment of endocrine disrupting compounds at the wastewater treatment works in Pietermaritzburg (South Africa). Sci. Total Environ. 2014 Jan;468-469:584-97.

14. Lee HB, Liu D. Degradation of $17 \beta$-estradiol and its metabolites by sewage bacteria. Water Air Soil Pollution. 2002;134:353-68.

15. E4876 SIGMA 17a-Ethynylestradiol [Internet]. [citado 2017 Ago 28]. Disponível em: http:// www.sigmaaldrich.com/catalog/product/sigma/e4876?lang=pt\&region=BR\#

16. Anwer F, Chaurasia S, Khan AA. Hormonally active agents in the environment: a state-of-the-art review. Rev Environ Health. 2016 Dec;31(4):415-33. 
17. Aquino SF, Brandt EMF, Chernicharo CAL. Remoção de fármacos e desreguladores endócrinos em estações de tratamento de esgoto: revisão da literatura. Eng. Sanit. Ambient. [Internet]. 2013 Set [citado 2017 Ago 30];18(3):187-204. Disponível em: http://www.scielo.br/scielo. php?script=sci_arttext\&pid=S1413-41522013000300187\&lng=en\&nrm=iso

18. Bhandari RK, Deem SL, Holliday DK, Jandegian CM, Kassotis CD, Nagel SC, et al. Effects of the environmental estrogenic contaminants bisphenol A and 17a-ethinyl estradiol on sexual development and adult behaviors in aquatic wildlife species. Gen Comp Endocrinol. 2015 Apr;214:195-219.

19. Green C, Brian J, Kanda R, Scholze M, Williams R, Jobling S. Environmental concentrations of anti-androgenic pharmaceuticals do not impact sexual disruption in fish alone or in combination with steroid oestrogens. Aquat Toxicol. 2015 Mar;160:117-27.

20. Oropesa AL, Jiménez B, Gil MC, Osswald J, Fallola C, Pula HJ, et al. Histological alterations in the structure of the testis in tench (Tinca tinca) after exposure to 17 alpha-ethynylestradiol. Environ Toxicol. 2014 Oct;29(10):1182-92.

21. Hu S, Zhang H, Shen G, Yuan Z, Xu T, Ji R. Effects of $17 \beta$-estradiol and $17 \alpha$-ethinylestradiol on the embryonic development of the clearhead icefish (Protosalanx hyalocranius). Chemosphere. 2017 Jun;176:18-24.

22. Sun M, Xu D, Ji Y, Liu J, Ling W, Li S, et al. Using Fenton Oxidation to Simultaneously Remove Different Estrogens from Cow Manure. Saúde Pública Int J Environ Res. 2016 Set;13(9).

23. Borek-Dohalska L, Klusonova Z, Holecova J, Martinkova M, Barta F, Dracinska H, et al. Exposure of rats to exogenous endocrine disruptors 17alpha-ethinylestradiol and benzo(a)pyrene and an estrogenic hormone estradiol induces expression of cytochromes P450 involved in their metabolism. Neuro Endocrinol Lett. 2016 Dec;18;37(1 Suppl):84-94.

24. Pereira RO, Postigo C, Alda ML, Daniel LA, Barcelo D. Removal of estrogens through water disinfection processes and formation of by-products. Chemosphere. 2011 Feb;82(6):789-99.

25. Li YM, Zeng QL, Yang SJ. Removal and fate of estrogens in an anaerobic-anoxic-oxic activated sludge system. Water Science and Technology. 2011 Jan;63:51-6.

26. Hamid H, Eskicioglu C. Fate of estrogenic hormones in wastewater and sludge treatment: a review of properties and analytical detection techniques in sludge matrix. Water Research. 2012;46:5813-33.

27. Racz LA, Goel RK. Fate and removal of estrogens in municipal wastewater. J. Environ. Monit. 2009;12:58-70.

28. Ting YF, Praveena SM. Sources, mechanisms, and fate of steroid estrogens in wastewater treatment plants: a mini review. Environ. Monit. Assess. 2017;189:178. 
29. Giowanella M, Bozza A, Dalzoto PR, Dionísio JA, Andraus S, Guimarães EL, et al. Microbiological quality of water from the rivers of Curitiba, Paraná State, Brazil, and the susceptibility to antimicrobial drugs and pathogenicity of Escherichia coli. Environ. Monit. Assess. 2015;187:673.

30. Nunes CN, Pauluk LE, Felsner ML, dos Anjos VE, Quináia SP. Rapid Screening Method for Detecting Ethinyl Estradiol in Natural Water Employing Voltammetry. J Anal Methods Chem. $2016 ; 2016$.

31. Torres NH, Américo JHP; Ferreira LFR; Nazato C, Maranho LA, Vilca FZ, et al. Fármacos no ambiente: revisão. REA - Revista de Estudos Ambientais. 2012;14(2):67-75.

32. Park CM, Heo J, Yoon Y. Oxidative degradation of bisphenol A and 17a-ethinyl estradiol by Fenton-like activity of silver nanoparticles in aqueous solution. Chemosphere. 2017 Feb;168:617-22.

33. Karim S, Bae S, Greenwood D, Hanna K, Singhal N. Degradation of 17a-ethinylestradiol by nano zero valent iron under different $\mathrm{pH}$ and dissolved oxygen levels. Water Res. 2017 Aug;125:32-41.

34. Jarošová B, Filip J, Hilscherová K, Tuček J, Šimek Z, Giesy JP, et al. Can zero-valent iron nanoparticles remove waterborne estrogens? J. Environ. Gerenciar. 2015 Mar;150:387-92.

35. Oliveira HG, Ferreira LH, Bertazzoli R, Longo C. Remediation of 17- $\alpha$-ethinylestradiol aqueous solution by photocatalysis and electrochemically-assisted photocatalysis using $\mathrm{TiO} 2$ and $\mathrm{TiO} 2 /$ WO3 electrodes irradiated by a solar simulator. Water Research. 2015 Apr;72(1):305-14.

36. Feng Y, Wang C, Liu J, Zhang Z. Electrochemical degradation of 17-alpha-ethinylestradiol (EE2) and estrogenic activity changes. J. Environ. Monit. 2010 Feb;12(2):404-8.

37. Wang Y, Li Y, Zhang W, Wang Q, Wang D. Photocatalytic degradation and reactor modeling of 17 a-ethynylestradiol employing titanium dioxide-incorporated foam concrete. Environ. Sci. Pollut. Res. Int. 2015 Mar;22(5):3508-17.

38. Unicef. Unicef e OMS lançam relatório sobre diarreia, a segunda maior causa de mortalidade infantil [Internet]. 2009. [citado 2017 Ago 25]. Disponível em: http://www.unicef.org/brazil/pt/ media_16165.htm

39. Augusto LGS, Gurgel IGD, Neto HFC, Melo CH, Costa AM. O contexto global e nacional frente aos desafios do acesso adequado à água para consumo humano. Ciência \& Saúde Coletiva. 2012;17(6):1511-22.

40. Yang Y, Li J, Lu K, Shi H, Gao S. Transformation of 17a-ethinylestradiol by simultaneous photoenzymatic process in Humic water. Chemosphere. 2017 Jul; 178:432-8.

Data da submissão: 03 de outubro de 2017 Avaliado em: 16 de outubro de 2017 (AVALIADOR A) Avaliado em: 27 de outubro de 2017 (AVALIADOR B) Aceito em: 28 de novembro de 2017 